\title{
miR-1908 as a novel prognosis marker of glioma via promoting malignant phenotype and modulating SPRY4/RAF1 axis
}

\author{
ZHI CHAI $^{1 *}$, HUIJIE FAN $^{1 *}$, YANYAN LI $^{1}$, LIJUAN SONG $^{1}$, XIAOMING JIN ${ }^{2}$, \\ JIEZHONG YU ${ }^{3}$, YANHUA $\mathrm{LI}^{3}$, CUNGEN MA ${ }^{1,3}$ and RAN ZHOU ${ }^{1}$ \\ ${ }^{1}$ Basic Medical College/2011 Collaborative Innovation Center/Neurobiology Research Center, \\ Shanxi University of Traditional Chinese Medicine, Jinzhong, Shanxi 030619, P.R. China; ${ }^{2}$ Stark Neuroscience \\ Research Institute, Indiana University School of Medicine, Indiana University, Indianapolis, IN 46202, USA; \\ ${ }^{3}$ Institute of Brain Science, Shanxi Datong University, Datong, Shanxi 037009, P.R. China
}

Received April 5, 2017; Accepted September 4, 2017

DOI: 10.3892/or.2017.6003

\begin{abstract}
MicroRNAs (miRNAs) are reported to be involved in the development of glioma. However, study on miRNAs in glioma is limited. The present study aimed to identify miRNAs which can act as potential novel prognostic markers for glioma and analyze its possible mechanism. We show that miR-1908 correlates with shorter survival time of glioma patients via promoting cell proliferation, invasion, anti-apoptosis and regulating SPRY4/RAF1 axis. Analysis of GEO and TCGA database found that miR-1908 was significantly upregulated in glioma tissues, and strongly associated with shorter survival time of glioma patients. Further Gene Ontology (GO) and Kyoto Encyclopedia of Genes and Genomes (KEGG) analysis revealed that miR-1908 is mainly involved in regulating cell proliferation, invasion and apoptosis. To further confirm the above results, in vitro, glioma U251 cells were transfected with miR-1908 mimics or inhibitor, and upregulated miR-1908 promoted U251 cell proliferation, and enhanced the ability of invasion by Transwell assay. In addition, upregulated miR-1908 also enhanced anti-apoptosis ability of U251 cells through decreasing pro-apoptosis protein Bax expression. Since miRNAs regulate numerous biological processes by
\end{abstract}

Correspondence to: Professor Cungen Ma or Professor Ran Zhou, Basic Medical College/2011 Collaborative Innovation Center, Shanxi University of Traditional Chinese Medicine, No. 121 University Street, Higher Education Park, Jinzhong, Shanxi 030619, P.R. China E-mail: macungen2001@163.com

E-mail: zhour58@sohu.com

${ }^{*}$ Contributed equally

Abbreviations: SPRY4, sprouty RTK signaling antagonist 4; RAF1, Raf-1 proto-oncogene, serine/threonine kinase; MMP-2/9, matrix metalloproteinase $-2 / 9$; BAX, BCL2 associated X protein

Key words: miR-1908, glioma, prognostic marker, SPRY4, RAF1, apoptosis targeting broad set of messenger RNAs, validated target genes of miR-1908 in glioma were analyzed by TargetScan and miRTarBase databases. Among them SPRY4 was significantly decreased in glioma tissues and associated with short survival time, which was selected as the key target gene of miR-1908. Moreover, protein-protein interaction (PPI) showed that SPRY4 could interacted with pro-oncogene RAF1 and negatively correlated with RAF1 expression. Consistent with above analysis, in vitro, western blot analysis identified that miR-1908 upregulated significantly decreased SPRY4 expression and increased RAF1 expression. Hence, miR-1908 was correlated with poor prognosis of glioma via promoting cell proliferation, invasion, anti-apoptosis and regulating SPRF4/ RAF1 axis. Our results elucidated the tumor promoting role of miR-1908 and established miR-1908 as a potential novel prognostic marker for glioma.

\section{Introduction}

Glioma is one of the most common primary intracranial malignant tumors in adults (1), and survival time after diagnosis is approximately one year. The profuse infiltration of glioma cells into healthy tissue surrounding the main tumor mass is one of the major obstacles limiting the improvement of patient survival (2). The incidence and mortality rate of glioma still continues to increase (3). Although many genetic and epigenetic changes were found to be related to glioma, the pathogenesis remains poorly understood.

miRNAs are a class of small molecular non-coding regulatory RNAs, which act as oncogenes or tumor suppressors in a variety of tumors $(4,5)$. Systematic and integrative analysis could detect key miRNAs that contribute to cancer development (6). The abnormal expression of miRNAs involved in transcriptional regulation network leads to tumor initiation and progression. Its main mechanism includes one-to-many and many-to-one regulation between transcription factor to miRNA and miRNA to target gene, which increases the complexity of miRNA regulation, thus, affecting the biological behavior of the tumor (7). Hence, there is considerable interest in using deregulated miRNAs as prognosis prediction markers and understanding their molecular targets for cancer treatment. 
During glioma occurrence and progression, abnormal expression of miRNAs was found to exert important functions. miR-182, miR-199b and miR-203 were upregulated (8-10), while miR-451, miR-17 and miR-184 were downregulated in glioma tissues $(11,12)$. These miRNAs were also reported to be involved in the development of glioma. However, miRNAs influencing glioma occurrence and progression is far from completely investigated, and no miRNA marker is used in clinic. Thus, there is still a growing need for developing prognostic markers and identifying therapeutic targets for improving the outcomes of glioma patients.

In the present study, we found that miR-1908 was significantly upregulated in glioma tissues. High level of miR-1908 was correlated with the shorter survival time of glioma, and promoted glioma cell proliferation and invasion and suppressed apoptosis. miR-1908 exerted these functions by regulating SPRY4/RAF1 axis and changing the expression of apoptosis related proteins $\mathrm{Bcl}-2 / \mathrm{Bax}$ and matrix metallopoteinase-2 (MMP-2). These results help to elucidate the pathogenesis and predict prognosis of glioma.

\section{Materials and methods}

Bioinformatics analysis. To identify the expression of miRNAs in primary human glioma and normal cerebral tissue, data about miRNA expression level in glioma patients were obtained from Gene Expression Omnibus (GEO) database (https://www.ncbi.nlm.nih.gov/gds/). Clinical data such as overall survival (OS), disease-free survival (DFS), lymph node metastasis, TNM stage and miR-1908 expression level were extracted from the Cancer Genome Atlas (TCGA) database (https://cancergenome.nih.gov/). Target genes of miR-1908 were analyzed by TargetScan (http://www.targetscan.org/) and miRTarBase database (experimentally validated miRNAtarget interactions database, http://mirtarbase.mbc.nctu.edu. tw/). Enrichment analysis was performed by cBioPortal online database (http://www.cbioportal.org/). The pathological images and IHC data of glioma were downloaded from the Human Protein Atlas web portal (www.proteinatlas.org), the sum IOD was analyzed by Image-Pro Plus software (version 6.0; Media Cybernetics, Inc., Rockville, MD, USA).

Gene Ontology and KEGG pathway analyses. To explore the functional annotation enrichment of the target genes of miR-1908 in glioma, GO and KEGG analyses were conducted using the Database for Annotation, Visualization and Integrated Discovery (DAVID) online analysis tool (https:// david.ncifcrf.gov/). The database provides a comprehensive set of functional annotation tools for investigators to understand biological meaning behind large list of genes, especially for identifying enriched biological themes.

Cell culture and miRNA transfection. Human glioma cell line U251 was purchased from the Cell Resource Center of Beijing Xiehe (Beijing, China) and cultivated in an incubator at $37^{\circ} \mathrm{C}$ with $5 \% \mathrm{CO}_{2}$. U251 cells were maintained in high-glucose Dulbecco's modified Eagle's medium (DMEM; Gibco, Waltham, MA, USA) supplemented with $10 \%$ fetal bovine serum (FBS; HyClone Laboratories, Inc., Logan, UT, USA) as well as penicillin (100 U/ ml; Thermo Fisher Scientific, Waltham, MA, USA).
miR-1908 mimics, miR-1908 inhibitor and their negative control (NC) sequences were designed and synthesized by Suzhou GenePharma Co., Ltd. (Shanghai, China). Lipofectamine 2000 (Invitrogen, Carlsbad, CA, USA) was used for miRNA oligo transfection according to the manufacturer's protocol. After $48 \mathrm{~h}$ of transfection, cells were used for the following experiments.

RNA isolation and quantitative real-time PCR assay $(q R T-P C R)$. Total RNA was extracted from U251 cells transfected with miR-1908 mimics or inhibitor using the TRIzol reagent (Invitrogen) according to the manufacturer's protocol. RNA quantity and purity were assessed using NanoDrop ND-1000 (Thermo Fisher Scientific). Total RNA was reversetranscribed to cDNA, and the first-strand cDNA was used as a template for real-time PCR (13). Reactions of qRT-PCR was done on the ABI Prism 7500 (Applied Biosystems, Foster City, CA, USA) using the commercially available gene expression assay for miR-1908, SPRY4 and RAF1. All reactions were run in triplicate. Relative gene expression was quantified using U6 or GAPDH as an internal control.

CCK- 8 assay. Cells transfected with miR-1908 mimics or inhibitor sequence were plated at a density of $5 \times 10^{3}$ cells/well onto 96-well plates at $37^{\circ} \mathrm{C}$ in an incubator with $5 \% \mathrm{CO}_{2}$. Cell proliferation was then assessed every $24 \mathrm{~h}$ using Cell Counting kit-8 (CCK-8; Sigma Aldrich St. Louis, MO, USA) according to standard protocol. For each sample at each time-point, six wells were analyzed. The experiment was repeated three times.

Cell invasion. Cell invasion abilities were determined in Transwell assays. Briefly, to determine the invasion potential, DMEM containing 10\% FBS was added to the lower chambers, and $1 \times 10^{5}$ cells suspended in $200 \mu 1$ serum-free medium were added to the upper chambers with Matrigel matrix gel and cultured for $24 \mathrm{~h}$. Finally, the cells that traversed the membrane were stained with crystal violet and counted.

Gelatin zymography assay. Activities of MMP-2 and MMP-9 were assessed by gelatin zymography assay. U251 cells were cultured for $24 \mathrm{~h}$ after transfecting the miR-1908 mimics or inhibitor. At the end of incubation, $40 \mu 1$ of culture supernatant was mixed with sample buffer and resolved on a $10 \%$ SDS-PAGE under non-reducing conditions. The gel was co-polymerized containing $0.5 \mathrm{mg} / \mathrm{ml}$ of gelatin (SigmaAldrich). Gel was washed twice for $30 \mathrm{~min}$ with renaturation buffer (2.5\% Triton X-100) at room temperature before incubation in the incubation buffer $(50 \mathrm{mM}$ Tris- $\mathrm{HCl} \mathrm{pH} \mathrm{7.5,200} \mathrm{mM}$ $\mathrm{NaCl}, 10 \mathrm{mM} \mathrm{CaCl}{ }_{2}, 1 \mu \mathrm{M} \mathrm{ZnCl}_{2}$ ) at $37^{\circ} \mathrm{C}$ for $36 \mathrm{~h}$. Thereafter, gel was stained for $2 \mathrm{~h}$ in $0.25 \%$ Coomassie brilliant blue R-250 and then de-stained. White bands were observed against a blue background after de-staining, indicating gelatinolytic activities of MMP-2 and MMP-9.

Western blotting. Cells were collected and lysed in lysis buffer (Beijing CoWin Biotech Co., Ltd., Beijing, China) in the presence of protease inhibitors for $30 \mathrm{~min}$ to extract total protein from cells transfected with miR-1908 mimics or inhibitor, and protein levels were quantified using bicinchoninic acid assays (Beijing CoWin Biotech). Subsequently, $30 \mu \mathrm{g}$ protein from 
Table I. The main dysregulated miRNAs in glioma tissue.

\begin{tabular}{clccc}
\hline No. & \multicolumn{1}{c}{ Name } & $\log _{2}$ fold change & Average expression & P-value \\
\hline 1 & hsa-miR-129-1-3p & -1.182734902 & 1.36366824 & 0.026579 \\
2 & hsa-miR-6749-5p & 1.063017222 & 6.664388833 & 0.027495 \\
3 & hsa-miR-6789-5p & 1.538438528 & 6.707455986 & 0.027804 \\
4 & hsa-mir-6800 & 1.041350944 & 8.824345889 & 0.027867 \\
5 & hsa-miR-4310 & 1.023917706 & 2.157122464 & 0.028345 \\
6 & hsa-mir-628 & -1.084536185 & 1.309829702 & 0.029722 \\
7 & hsa-miR-1908-5p & 1.031185667 & 8.369524444 & 0.030411 \\
8 & hsa-miR-7977 & -1.52912775 & 7.489592208 & 0.033013 \\
9 & hsa-miR-1238-3p & 1.018972278 & 1.632209472 & 0.033941 \\
10 & hsa-miR-3162-5p & 1.320415407 & 5.361444565 & 0.033947 \\
11 & hsa-mir-5095 & 1.224927278 & 4.761240056 & 0.036548 \\
12 & hsa-miR-6503-3p & 1.830256801 & 2.51717765 & 0.03752 \\
13 & hsa-miR-4767 & 1.9436247 & 2.648728206 & 0.037793 \\
14 & hsa-miR-8089 & 1.274433444 & 4.688330611 & 0.039185 \\
15 & hsa-miR-6724-5p & 1.074298778 & 9.620908778 & 0.043217 \\
\hline
\end{tabular}

each sample was loaded onto $10 \%$ sodium dodecyl sulfate (SDS) polyacrylamide gels and subjected to SDS-polyacrylamide gel electrophoresis (PAGE; Beijing CoWin Biotech). Protein was then transferred to nitrocellulose membranes (Sigma-Aldrich), which were then blocked with bovine serum albumin buffer (BSA; Invitrogen) for $1 \mathrm{~h}$. Membranes were then incubated with primary antibodies targeting SPRY4 (1:1,000 dilution, cat. no. ab59785; Abcam), RAF1 (1:1,000 dilution, cat. no. ab154754; Abcam), Bax (1:2,000 dilution, cat. no. ab182733; Abcam), Bcl-2 (1:1,000 dilution, cat. no. ab194583; Abcam) or GAPDH (1:5,000 dilution, cat. no. ab70699; Abcam) overnight at $4^{\circ} \mathrm{C}$, followed by incubation with goat anti-rabbit horseradish peroxidase (HRP)-conjugated secondary antibody (1:3,000 dilution, cat. no. CW0103; Beijing CoWin Biotech) for $1 \mathrm{~h}$ at room temperature. Detection was facilitated using an enhanced chemiluminescence kit and images were analyzed using ImageJ software (version 1.62; National Institutes of Health, Bethesda, MD, USA).

Annexin V-FITC/PI apoptosis detection. Annexin V-FITC/PI (BD Biosciences, Bedford, MA, USA) was used to detect cell apoptosis rate. In brief, U251 cells transfected with miR-1908 mimics or inhibitor were washed twice with cold phosphatebuffered saline (PBS), and cells were added in $400 \mu \mathrm{l}$ binding buffer (provided by the manufacturer) at a concentration of $1 \times 10^{6}$ cells $/ \mathrm{ml}$. Annexin V-FITC (5 $\left.\mu \mathrm{l}\right)$ and PI (5 $\left.\mu \mathrm{l}\right)$ were then added, and the cells were analyzed with a flow cytometer (BD Accuri C6; BD Biosciences) within 1 h (13).

Protein-protein interaction (PPI) network construction. Search Tool for the Retrieval of Interacting Genes/Proteins (STRING; Search Tool for the Retrieval of Interacting Genes, http://string-db.org/) is a database of known and predicted protein interactions that may aid in the comprehensive description of cellular mechanisms and functions. The PPI network of the selected target genes of miR-1908 in glioma was constructed using the STRING database.
Statistical analysis. Statistical analyses were performed using the SPSS 21.0 (SPSS, Inc., Chicago, IL, USA) and GraphPad Prism (GraphPad Software, Inc., San Diego, CA, USA). GEO microarray data and TCGA data were analyzed by paired sample t-test. The log-rank test for the generated KaplanMeier (K-M) curve was conducted to evaluate the association between the expression level of miR-1908 and the survival rate. OS was defined as the time between the first surgery for primary glioma and death for any reason. DFS was defined as the time between the first surgical resection and disease recurrence. Growth curves were analyzed using a repeated measures ANOVA followed by Bonferroni post hoc analysis. Cell invasion data, apoptosis data and IHC data were analyzed by independent samples t-test. The correlation of target genes of miR-1908 expression level was analyzed by the Pearson correlation analysis. $\mathrm{P}<0.05$ was considered to indicate a statistically significant difference.

\section{Results}

Increased expression of miR-1908 in glioma and its association with shorter survival time in the patients. We used GEO microarray and TCGA miRNA dataset to screen miRNAs which related to glioma occurrence and development. Three pairs of glioma tissues and adjacent normal cerebral tissues were compared by miRNA microarray, 53 differentially expressed miRNAs $(\mathrm{P}<0.05 ; \mathrm{FC}>1$ and $\mathrm{FC}<-1)$ were identified. Of which, miR-1908 was one of rarely reported miRNAs in glioma and attracted our interest (Fig. 1A and Table I).

To further validate the abnormal regulated expression level of miR-1908, we compared the expression level of miR-1908 in glioma tissue and normal tissue by GEO database. Consistent with previous reports, miR-1908 was significantly upregulated in tumor tissue specimens $(n=89)$ compared with matched normal controls $(n=32)$ (Fig. $1 B ; P=0.0343)$. To further determine the predictive value of miR-1908 expression level as prognostic marker, 206 glioma patients from TCGA database 


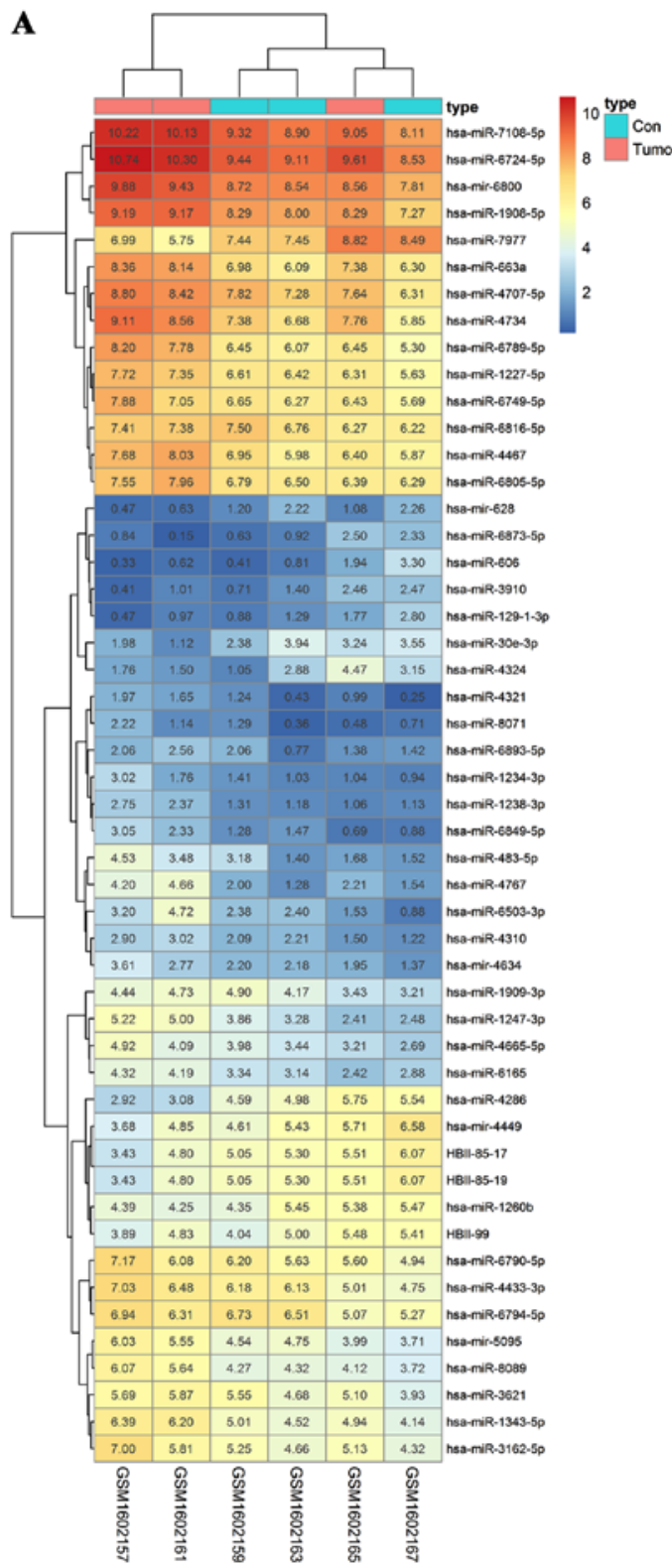

B

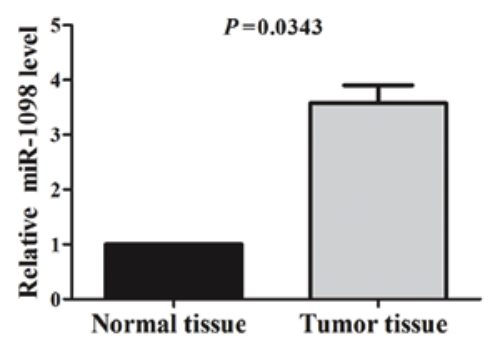

C

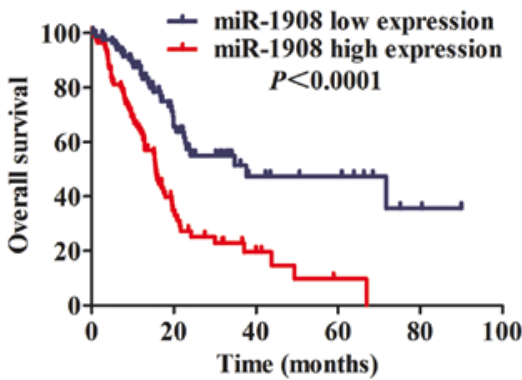

D

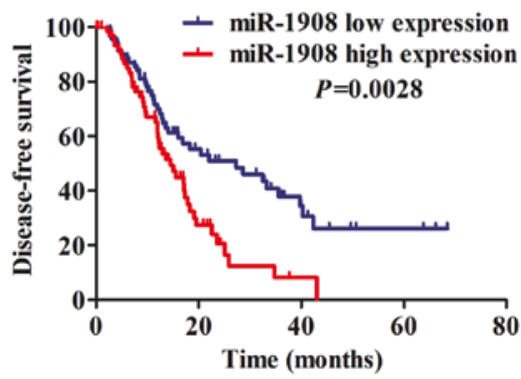

Figure 1. Expression level of miR-1908 in glioma tissues is upregulated. (A) Screening of differentially expressed genes in glioma based on GEO database. (B) The miR-1908 level was upregulated in glioma tissues $(\mathrm{n}=89)$ compared with adjacent normal tissues $(\mathrm{n}=32)$ by GEO analysis. (C) K-M curve for overall survival (OS) of patients with high ( $\mathrm{n}=103)$ and low $(\mathrm{n}=103)$ miR-1908 expression level. Results revealed that high miR-1908 expression level was correlated with shorter OS for glioma patients. (D) Patients with high miR-1908 expression ( $\mathrm{n}=103)$ had shorter disease-free survival (DFS) time than those with low miR-1908 expression $(n=103)$.

were divided into miR-1908 high expression and miR-1908 low expression groups according to median miR-1908 expression level. K-M curve showed that miR-1908 high expression group had shorter OS and DFS time than miR-1908 low expression group in terms of survival duration (Fig. $1 C$ and $\mathrm{D} ; \mathrm{P}<0.0001$ and $\mathrm{P}=0.0028$, respectively), particularly in overall survival. The result suggests that upregulated miR-1908 may be a novel prognostic marker for glioma patients.

miR-1908 is involved in multiple types of cancer-related pathways. In order to understand the function and role of miR-1908 in tumor progression, GO and KEGG pathway analysis were performed. We noted that these genes were especially enriched in functions of regulating cell proliferation, invasion and apoptosis (Fig. 2A). miRNAs were reported to promote cancer occurrence and progression through activating related signaling pathways. To better understand underlying molecular mechanisms in which miR-1908 plays roles, we investigated miR-1908 relevant KEGG pathways. Results revealed that miR-1908 was involved in 54 significant KEGG pathways, the main signaling pathways are shown in Fig. 2B. These pathways mainly included multiple types of cancer-related signaling pathways, especially the Jak-STAT, MAPK and Ras signaling pathways.

miR-1908 promotes cell proliferation and invasion of glioma. In order to demonstrate the effect of miR-1908 on glioma malignant phenotype, we performed cell proliferation and invasion assays. qRT-PCR results reveal that relative miR-1908 level was upregulated in U251 cells transfected 


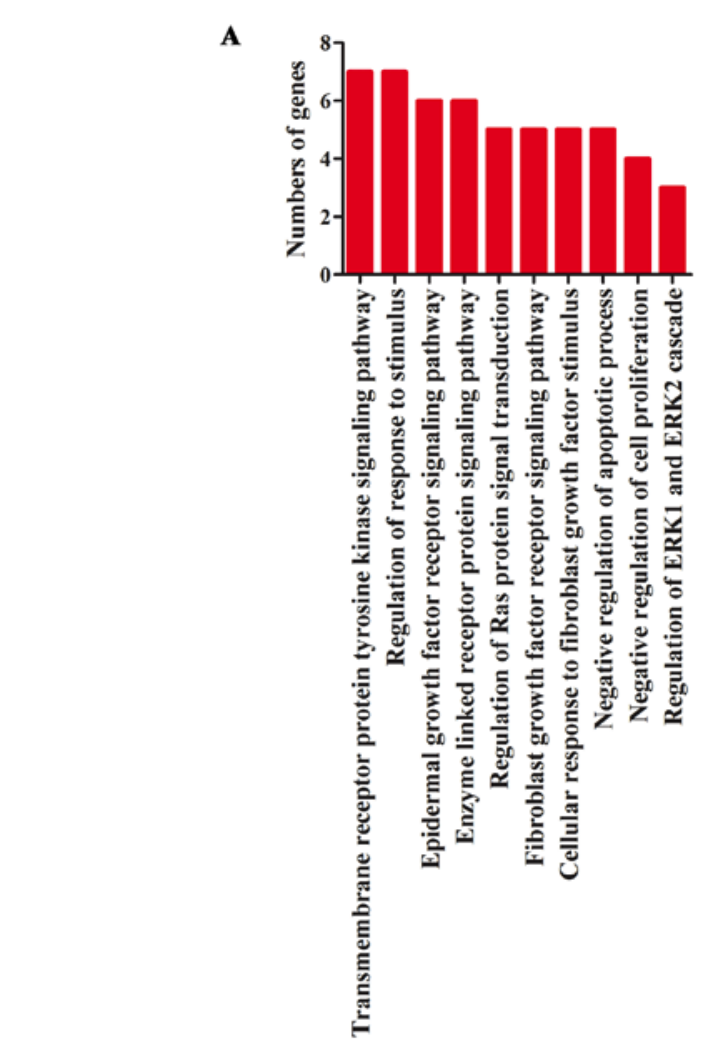

B

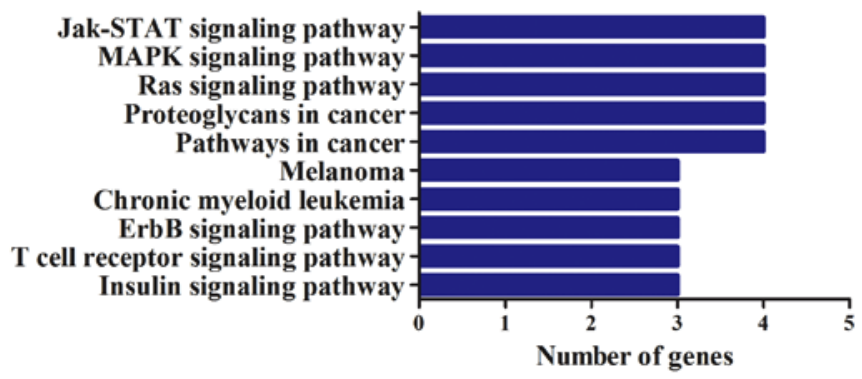

Figure 2. miR-1908 is involved in multiple types of cancer-related pathways. (A) GO analysis of the miR-1908 according to biological process, molecular function and cellular component. (B) miR-1908 was involved in 10 significant signaling pathways.

with miR-1908 mimics (Fig. 3A; P=0.0239), and miR-1908 level was downregulated after miR-1908 inhibitor transfection (Fig. 3B; $\mathrm{P}=0.0210$ ). The ability of miR-1908 to modulate the proliferation of U251 cells was analyzed using a CCK-8 assay. The results indicated that downregulated miR-1908 could inhibit cell proliferation in 72 and $96 \mathrm{~h}$ (Fig. 3C; $\mathrm{P}=0.0285$ and $\mathrm{P}=0.0152$, respectively), while upregulated miR-1908 significantly enhanced the ability of cell proliferation (Fig. 3D; $\mathrm{P}=0.0254$ and $\mathrm{P}=0.0196$, respectively). Similarly, upregulated miR-1908 also significantly promoted cell invasion compared with control group through Transwell assay, on the contrary, downregulated miR-1908 in U251 cells inhibited invasion (Fig. 3E and F; $\mathrm{P}=0.026$ and $\mathrm{P}=0.030$, respectively).

Previous studies have shown that proteolysis is a necessary part of the invasion process (14), and that increased expression of several members of the MMP family are correlated with high-grade gliomas, especially MMP-2/9 (15). We therefore detected the activity of MMP-2/9 by using gelatin zymography assay. The results revealed that upregulated miR-1908
Table II. The main target genes of miR-1908.

\begin{tabular}{|c|c|c|c|c|}
\hline No. & $\begin{array}{l}\text { Gene } \\
\text { name }\end{array}$ & $\begin{array}{l}\text { Cumulative } \\
\text { weighted } \\
\text { context score }\end{array}$ & $\begin{array}{c}\text { Total } \\
\text { context } \\
\text { score }\end{array}$ & $\begin{array}{c}\text { Aggregate } \\
\text { PCT }\end{array}$ \\
\hline 1 & PRSS22 & -1.51 & -1.51 & N/A \\
\hline 2 & TOR4A & -1.02 & -1.02 & N/A \\
\hline 3 & ZNF385A & -0.9 & -0.9 & N/A \\
\hline 4 & DPF1 & -0.9 & -0.9 & N/A \\
\hline 5 & SRCIN1 & -0.8 & -0.8 & N/A \\
\hline 6 & SCAMP4 & -0.79 & -0.79 & N/A \\
\hline 7 & RAB11B & -0.75 & -0.97 & N/A \\
\hline 8 & GNAI2 & -0.74 & -0.74 & N/A \\
\hline 9 & NRGN & -0.7 & -0.7 & N/A \\
\hline 10 & NKX2-5 & -0.7 & -0.78 & N/A \\
\hline 11 & H2AFX & -0.62 & -0.62 & N/A \\
\hline 12 & POU3F1 & -0.54 & -0.54 & N/A \\
\hline 13 & BARHL1 & -0.53 & -0.53 & N/A \\
\hline 14 & FAM83H & -0.49 & -0.49 & N/A \\
\hline 15 & SLC11A1 & -0.49 & -0.49 & N/A \\
\hline 16 & GRB2 & -0.45 & -0.45 & N/A \\
\hline 17 & CALR & -0.43 & -0.43 & N/A \\
\hline 18 & PRX & -0.42 & -0.42 & N/A \\
\hline 19 & POFUT2 & -0.41 & -0.41 & N/A \\
\hline 20 & TACC3 & -0.41 & -0.41 & N/A \\
\hline 21 & CASZ1 & -0.41 & -0.41 & N/A \\
\hline 22 & GPR20 & -0.4 & -0.48 & N/A \\
\hline 23 & SIGLEC12 & -0.4 & -0.4 & N/A \\
\hline 24 & MYADM & -0.38 & -0.45 & N/A \\
\hline 25 & SPRY4 & -0.38 & -0.47 & N/A \\
\hline 26 & SYNGR1 & -0.37 & -0.37 & N/A \\
\hline 27 & MARVELD1 & -0.36 & -0.36 & N/A \\
\hline 28 & PHF15 & -0.36 & -0.53 & N/A \\
\hline 29 & APOE & -0.33 & -0.33 & N/A \\
\hline 30 & NACC1 & -0.33 & -0.36 & N/A \\
\hline
\end{tabular}

expression significantly enhanced the activity of MMP-2, but there was no significant difference in MMP-9 activity between miR-1908 mimics group and control group (Fig. 3G and H).

miR-1908 inhibits cell apoptosis via regulating Bax/Bcl-2 expression. The processes of apoptosis, which induce degradation of proteins and organelles or cell death upon cellular stress, are crucial in the pathophysiology of various tumors. In order to observe the effect of miR-1908 overexpression on cell apoptosis, miR-1908 mimics or inhibitor were transfected into U251 cells, and apoptosis rate and the expression of apoptosisassociated protein $\mathrm{Bax} / \mathrm{Bcl}-2$ were detected by flow cytometry and western blot analysis, respectively. Representative results of flow cytometric analysis are shown in Fig. 4A and C. In general, the rates of cell death were low $(<5 \%)$. Apoptosis rate was not statistically different in cells transfected with miR-1908 mimics 
A

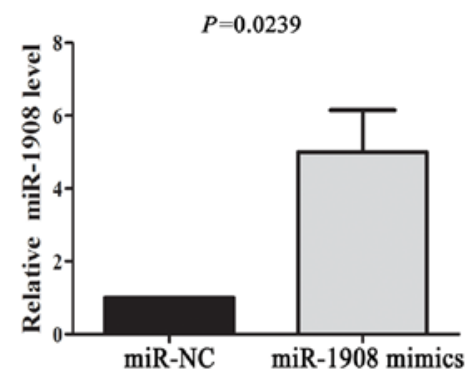

C

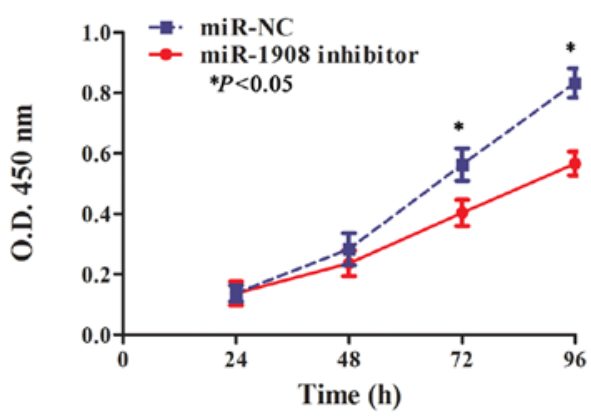

B

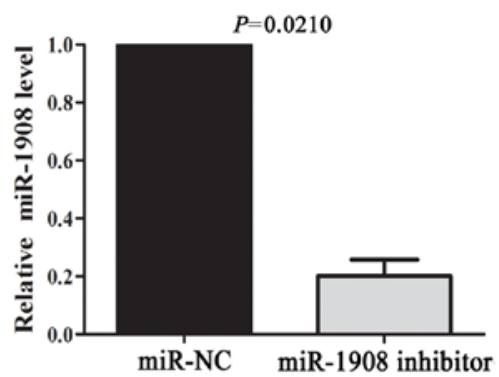

D

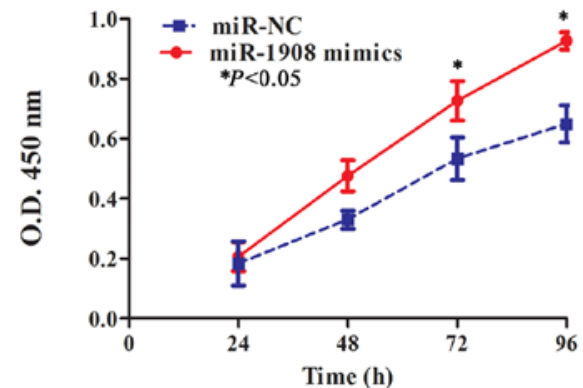

E

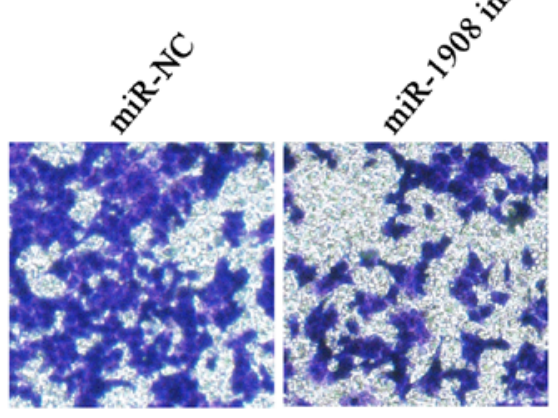

G
F

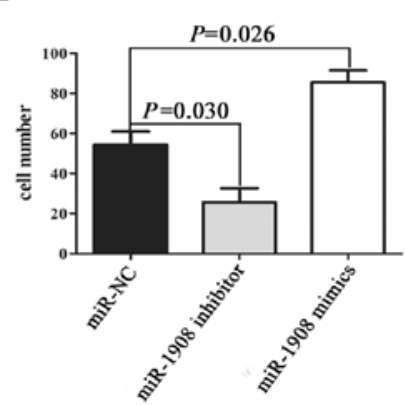

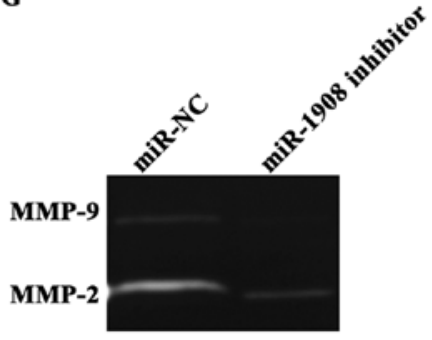

H

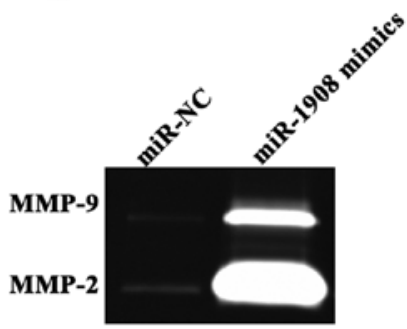

Figure 3. miR-1908 promotes glioma cell proliferation and invasion. (A and B) The expression of miR-1908 in U251 cell were affected by transfection of miR-1908 mimics or inhibitor. (C) Downregulated miR-1908 level inhibited the proliferation of U251 cells. (D) miR-1908 significantly promoted the proliferation of U251 cells by $>50 \%$ at all time-points following $72 \mathrm{~h}$ of incubation. (E and F) Upregulated miR-1908 enhanced invasion ability of glioma cells. (G and H) miR-1908 mimics enhanced the activity of MMP-2, while miR-1908 inhibitor reduced its activity.

and negative control sequences (Fig. 4B; $\mathrm{P}=0.083$ ). However, U251 cells transfected with miR-1908 inhibitor had significantly higher rate of apoptosis when compared with cells transfected with negative control sequences (Fig. 4D; P=0.027). Furthermore, transfection of miR-1908 inhibitor caused an increase in the expression of Bax, a pro-apoptotic protein, but a marked decrease in the expression of Bcl-2, an anti-apoptotic protein. In contrast, transfection of miR-1908 mimics downregulated the expression level of Bax. These data supported that miR-1908 enhances the ability of cell anti-apoptosis via regulating Bax/Bcl-2 expression.
SPRY4 is a key target gene of miR-1908 in glioma. miRNAs exert important functions in various biological processes via directly suppressing the expression of their target genes. According to integration analysis from TargetScan and miRTarBase database, 96 verified target genes (5) of miR-1908 were identified (Fig. 5A and Table II). Of which, SPRY4 was significantly downregulated in glioma tissue $(n=11)$ compared with normal tissue $(n=2)$ (Fig. $5 \mathrm{~B}$ and $\mathrm{C} ; \mathrm{P}=0.0054)$. Moreover, patients with SPRY4 alteration had lower OS and DFS compared with patients without alteration (Fig. 5D and E; 

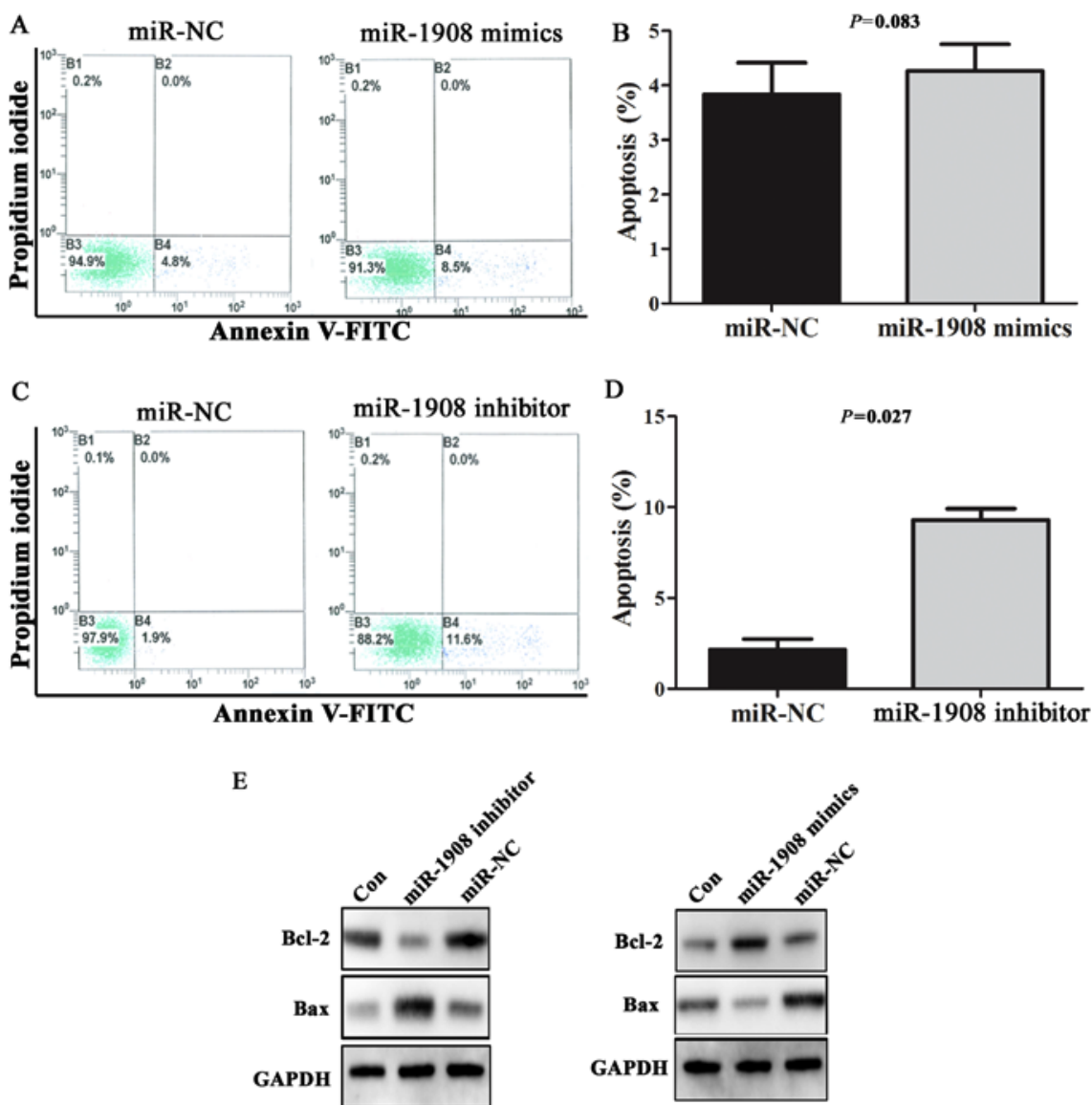

Figure 4. miR-1908 enhances the ability of glioma cell anti-apoptosis via regulating Bcl-2/Bax expression. (A-D) Double staining with Annexin V-FITC and PI was used to assess apoptosis in U251 cells transfected with miR-1908 mimics or inhibitor, and miR-1908 inhibitor markedly increased cell apoptosis rate. (E) Western blot analysis was performed to determine Bcl-2 and Bax protein expression in U251 cells transfected with miR-1908 mimics or inhibitor, and Bax was increased following transfection with miR-1908 inhibitor, but a marked decrease in the expression of Bcl-2. In contrast, transfection of miR-1908 mimics downregulated the expression level of Bax.

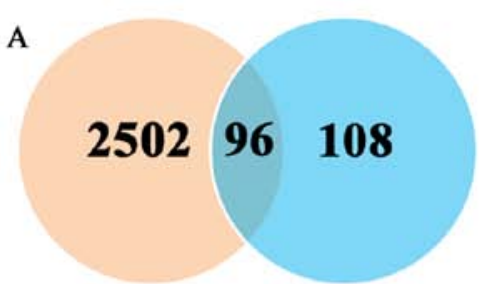

TargetScan miRTarBase
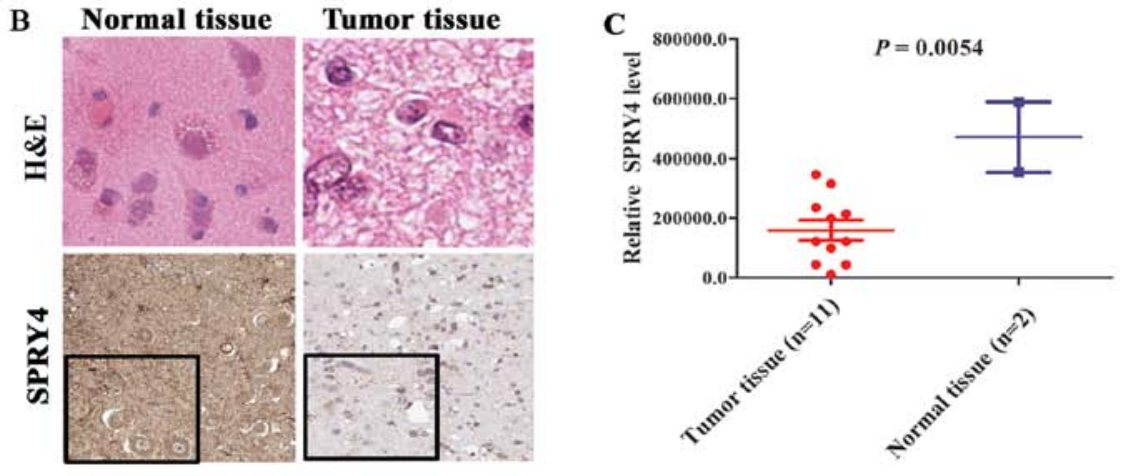
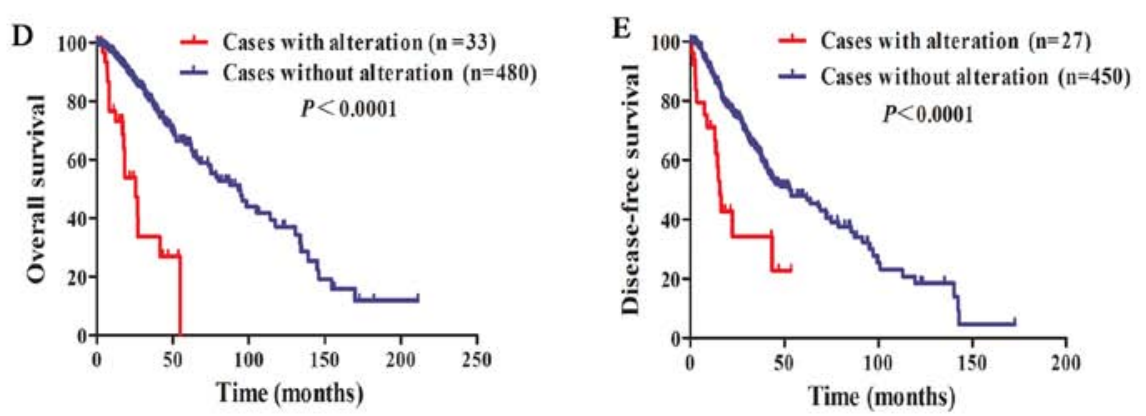

Figure 5. SPRY4 is a key target gene of miR-1908. (A) Target genes of miR-1908 were identified by TargetScan and miRTarBase database. (B and C) The Human Protein Atlas database results revealed that the expression level of SPRY4 was downregulated in glioma compared with normal tissues. (D) K-M curve for OS of patients with $(n=33)$ and without $(n=480)$ alteration. (E) Patients with SPRY4 alteration $(n=27)$ had shorter DFS than without alteration $(n=450)$. 
A

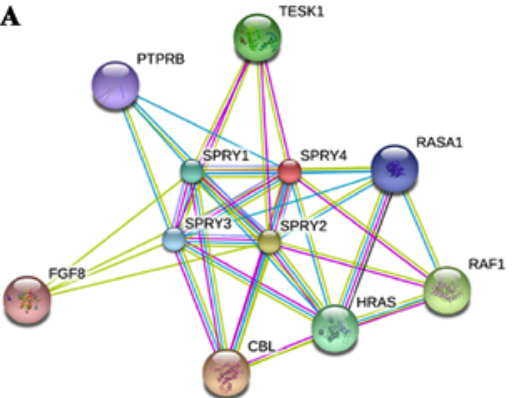

C

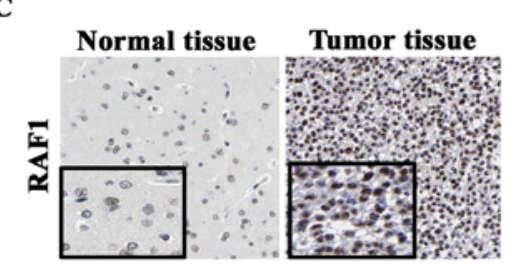

E

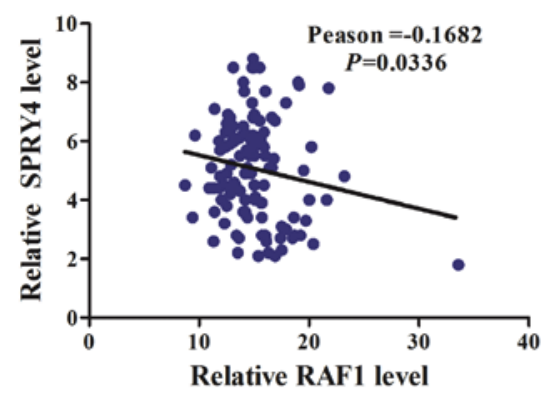

G

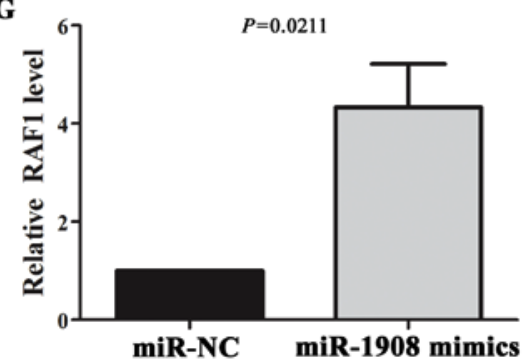

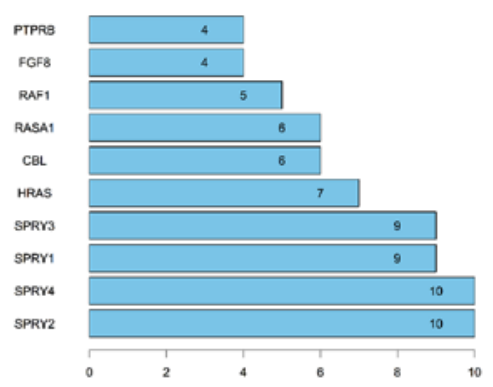

D

F
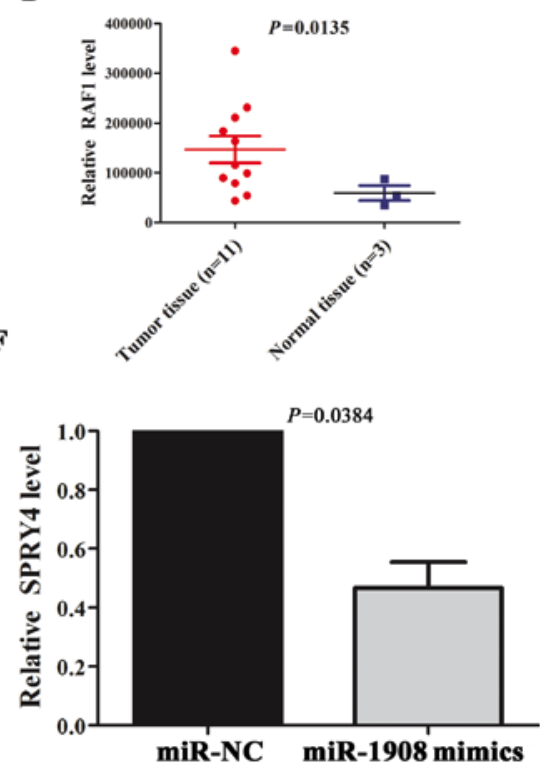

H

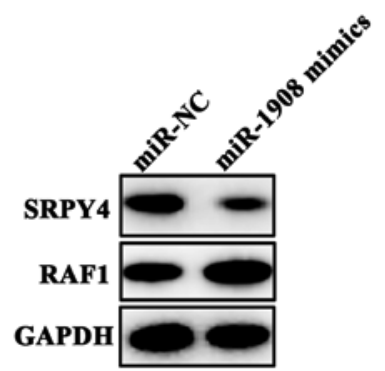

Figure 6. miR-1908 upregulates the expression level of RAF1 through targeting SPRY4. (A and B) PPI analysis of interaction genes with SPRY4, and interaction with pro-oncogene RAF1. (C and D) The expression level of RAF1 was upregulated in glioma tissue compared with normal tissue. (E) The expression level of SPRY4 was negatively correlated with RAF1 in glioma patients from TCGA KIRC database. (F and G) miR-1908 mimics were transfected into U251 cells, the relative mRNA level of SPRY4 and RAF1 were detected by qRT-PCR. (H) Upregulated miR-1908 promoted RAF1 expression via decreasing the expression of SPRY4.

$\mathrm{P}<0.0001)$. Hence, SPRY4 may be a key and specific target gene of miR-1908 in glioma.

SPRY4/RAF1 axis is regulated by miR-1908 in glioma. PPI analysis revealed that SPRY4 can interact with multiple proteins, included SPRY2, SPRY1, SPRY3, HRAS, CBL, RASA1, RAF1, FGF8 and PTPRB (Fig. 6A and B). Above all, RAF1 is a well-known pro-oncogene in multiple types of tumors (16). The abnormal upregulation of RAF1 protein plays an important role in the malignant transformation of tumor. In glioma tissues $(n=11), R A F 1$ protein expression levels was significantly upregulated (Fig. 6C and D; $\mathrm{P}=0.0135$ ) compared with normal tissues $(n=3)$. To further verify the relationship between SPRY4 and RAF1, correlation analyses between expression levels of SPRY4 and RAF1 were performed in glioma tissues. The results showed that the expression level of SPRY4 was negatively correlated with the expression level of RAF1 (Fig. 6E; Pearson=-0.1682, $\mathrm{P}=0.0336$ ). In addition, gene co-expression analysis revealed that RAF1 was significantly co-expressed with SPRY4 (Table III). To further validate whether miR-1908 could promote the expression of RAF1 via targeting SPRY4, miR-1908 mimics were transfected into U251 cells. qRT-PCR assay showed that SPRY4 mRNA level was downregulated in these cells, on the contrary, RAF1 mRNA level was significantly upregulated (Fig. 6F and G; P=0.0384 and $\mathrm{P}=0.0211$, respectively). Similarly, compared with negative group, the expression level of SPRY4 in U251 cells transfected with miR-1908 mimics was significantly downregulated and 
Table III. The co-expession genes with SPRY4 ranked by cBioPortal database.

\begin{tabular}{lcccc}
\hline Mean & $\begin{array}{c}\text { Mean } \\
\text { protein } \\
\text { expression } \\
\text { (Altered) }\end{array}$ & $\begin{array}{c}\text { expression } \\
\text { protein }\end{array}$ & & \\
\hline (Unaltered) & P-value & q-value \\
RAF1 & -0.11 & -0.44 & $9.61 \mathrm{E}-06$ & $1.39 \mathrm{E}-03$ \\
NRG1 & 0.13 & 0.33 & $1.21 \mathrm{E}-05$ & $1.39 \mathrm{E}-03$ \\
EIF4G1 & -0.31 & -0.61 & $2.93 \mathrm{E}-05$ & $2.24 \mathrm{E}-03$ \\
PRKAA1_PT172 & 0.25 & -0.05 & $1.06 \mathrm{E}-04$ & $3.55 \mathrm{E}-03$ \\
ARAF_PS299 & -0.13 & -0.03 & $1.15 \mathrm{E}-04$ & $3.55 \mathrm{E}-03$ \\
MYH11 & -1.75 & -0.8 & $1.26 \mathrm{E}-04$ & $3.55 \mathrm{E}-03$ \\
PGR & -0.1 & 0 & $1.30 \mathrm{E}-04$ & $3.55 \mathrm{E}-03$ \\
GATA3 & -0.52 & -0.28 & $1.35 \mathrm{E}-04$ & $3.55 \mathrm{E}-03$ \\
BAX & -0.1 & -0.51 & $1.40 \mathrm{E}-04$ & $3.55 \mathrm{E}-03$ \\
ADAR & -0.46 & -0.24 & $1.58 \mathrm{E}-04$ & $3.61 \mathrm{E}-03$ \\
RAB25 & -1.28 & -0.91 & $1.91 \mathrm{E}-04$ & $3.97 \mathrm{E}-03$ \\
IRS1 & -0.18 & 0.03 & $4.04 \mathrm{E}-04$ & $6.74 \mathrm{E}-03$ \\
ANXA7 & -0.02 & 0.17 & $4.88 \mathrm{E}-04$ & $6.74 \mathrm{E}-03$ \\
PARK7 & 0.12 & 0.37 & $5.00 \mathrm{E}-04$ & $6.74 \mathrm{E}-03$ \\
\hline
\end{tabular}

the expression level of RAF1 was increased (Fig. 6H). These data suggested that miR-1908 contributed to tumor progression through modulation of SPRY4/RAF1 axis in glioma.

\section{Discussion}

In the present study, integrative analysis of GEO and TCGA data suggested that the expression level of miR-1908 was significantly upregulated in glioma tissues and was associated with shorter survival time of glioma patients. Furthermore, we verified that miR-1908 could promote glioma cell proliferation, invasion, anti-apoptosis and regulate SPRY4/RAF1 axis. These results elucidated that miR-1908 is a novel prognosis marker via promoting malignant phenotype and modulating SPRY4/RAF1 axis. To the best of our knowledge, this is the first report showing prognostic value of miR-1908 for glioma patients.

A previous work demonstrated that miR-1908 was aberrantly expressed in several types of tumors, including osteosarcoma (17), lung (18) and liver cancer (19). miR-1908 is strongly associated with cell proliferation and migration (17), and poor prognosis of osteosarcoma patients (20). miR-1908 exerts these effects via regulating many signaling pathways of tumor formation. For example, overexpressed miR-1908 clusters downregulate the MARK1 signaling pathway to alter cell proliferation and differentiation in hepatoma cells, and serves as a biomarker for poor prognosis in osteosarcoma (20). miRNA-1908 functions as an oncogene in glioblastoma by repressing the PTEN signaling pathway (21). Notably, previous bioinformatics analysis also found that miR-1908 may contribute to glioma progression and be correlated with survival rate (22), but lacks sufficient experimental bases. In this study, we not only showed that miR-1908 acts as a novel prognostic marker for glioma, but also explored its possible mechanisms. Clinical data showed that high expression of miR-1908 was correlated with shorter OS and DFS. Upregulation of miR-1908 in vitro promoted glioma cell proliferation and invasion, and suppressed its apoptosis. These data indicated that miR-1908 contributes to poor prognosis of glioma patients via promoting glioma cell malignant behavior.

miRNAs play their roles via suppressing the expression of target mRNAs. Actually, the biological interactions between miRNAs and their targets are very complex in vivo. One miRNA may target multiple genes and target genes are tissue specific $(23,24)$. On the basis of known target gene database, we further combined TargetScan and miRTarBase datasets and used cBioPortal online analysis tool to find the tissue specific and key target genes of miR-1908 in glioma. Among them, SPRY4, a tumor suppressor, was downregulated in glioma patients and patients with SPRY4 alteration had shorter OS and DFS. Hence, SPRY4 was identified as a key target gene of miR-1908.

Our further analysis revealed that mainly ten proteins in glioma could interact and form the complex with SPRY4. Among these ten interacting proteins, RAF1 is a well-known pro-oncogene (25). In glioma tissue, SPRY4 expression level was significantly downregulated, but RAF1 expression level was significantly upregulated. The relationship between SPRY4 and RAF1 were further verified by correlation analysis of expression levels in tissues and in vitro. miR-1908 could suppress the expression of SPRY4 and upregulate RAF1 expression. Since proto-oncogene RAF1 serves as part of the mitogen-activated protein kinases/extracellular signalregulated kinase signal transduction pathway and regulates cell migration, apoptosis and differentiation (26), we speculate that miR-1908 may promote glioma progression through regulating SPRY4/RAF1 axis. Further experiments are necessary to verify our hypothesis.

In conclusion, miR-1908 is potentially a novel prognostic biomarker for glioma via promoting glioma cell proliferation, invasion, anti-apoptosis and regulating SPRY4/RAF1 axis. This study provides a novel prognostic marker and a new treatment direction for glioma.

\section{Acknowledgements}

The present study was supported by funding from the National Natural Science Foundation of China (no. 81102552), the International Science and Technology Cooperation Project of Shanxi Province (no. 2014081049-4), the Research Project Supported by Shanxi Scholarship Council of China (no. 2017-129), the Returned Chinese Scholars Technology Activities Preferred Project, Shanxi Province of China (no. 2017-19) and the Great Science and Technology Innovation Team Project of Traditional Chinese Medicine of Shanxi University (no. 20150401).

\section{References}

1. Lin L, Wang G, Ming J, Meng X, Han B, Sun B, Cai J and Jiang C: Analysis of expression and prognostic significance of vimentin and the response to temozolomide in glioma patients. Tumour Biol 37: 15333-15339, 2016.

2. Thompson EG and Sontheimer H: A role for ion channels in perivascular glioma invasion. Eur Biophys J 45: 635-648, 2016. 
3. Ho VK, Reijneveld JC, Enting RH, Bienfait HP, Robe P, Baumert BG and Visser O; Dutch Society for Neuro-Oncology (LWNO): Changing incidence and improved survival of gliomas. Eur J Cancer 50: 2309-2318, 2014.

4. Liang AL, Zhang TT, Zhou N, Wu CY, Lin MH and Liu YJ: MiRNA-10b sponge: An anti-breast cancer study in vitro. Oncol Rep 35: 1950-1958, 2016.

5. Chou CH, Chang NW, Shrestha S, Hsu SD, Lin YL, Lee WH, Yang CD, Hong HC, Wei TY, Tu SJ, et al: miRTarBase 2016: Updates to the experimentally validated miRNA-target interactions database. Nucleic Acids Res 44 (D1): D239-D247, 2016.

6. Zhu J, Wang S, Zhang W, Qiu J, Shan Y, Yang D and Shen B Screening key microRNAs for castration-resistant prostate cancer based on miRNA/mRNA functional synergistic network. Oncotarget 6: 43819-43830, 2015.

7. Zhao R, Zhou M, Wang H, Xiong W, Li X and Li G: MiRNA regulatory mechanism in tumor initiation and progression. Zhong Nan Da Xue Xue Bao Yi Xue Ban 38: 1282-1288, 2013 (In Chinese).

8. Garzia L, Andolfo I, Cusanelli E, Marino N, Petrosino G, De Martino D, Esposito V, Galeone A, Navas L, Esposito S, et al: MicroRNA-199b-5p impairs cancer stem cells through negative regulation of HES1 in medulloblastoma. PLoS One 4: e4998, 2009.

9. Costa FF, Bischof JM, Vanin EF, Lulla RR, Wang M, Sredni ST, Rajaram V, Bonaldo MF, Wang D, Goldman S, et al: Identification of microRNAs as potential prognostic markers in ependymoma PLoS One 6: e25114, 2011.

10. Jiang L, Mao P, Song L, Wu J, Huang J, Lin C, Yuan J, Qu L, Cheng SY and Li J: miR-182 as a prognostic marker for glioma progression and patient survival. Am J Pathol 177: 29-38, 2010.

11. Gal H, Pandi G, Kanner AA, Ram Z, Lithwick-Yanai G Amariglio N, Rechavi G and Givol D: MIR-451 and Imatinib mesylate inhibit tumor growth of Glioblastoma stem cells. Biochem Biophys Res Commun 376: 86-90, 2008.

12. Malzkorn B, Wolter M, Liesenberg F, Grzendowski M, Stühler K, Meyer HE and Reifenberger G: Identification and functional characterization of microRNAs involved in the malignant progression of gliomas. Brain Pathol 20: 539-550, 2010.

13. You BR, Shin HR, Han BR and Park WH: PX-12 induces apoptosis in Calu-6 cells in an oxidative stress-dependent manner. Tumour Biol 36: 2087-2095, 2015.

14. Deakin NE and Chaplain MA: Mathematical modeling of cancer invasion: The role of membrane-bound matrix metalloproteinases. Front Oncol 3: 70, 2013.
15. Fillmore HL, VanMeter TE and Broaddus WC: Membrane-type matrix metalloproteinases (MT-MMPs): Expression and function during glioma invasion. J Neurooncol 53: 187-202, 2001.

16. Ram RR, Mendiratta S, Bodemann BO, Torres MJ, Eskiocak U and White MA: RASSF1A inactivation unleashes a tumor suppressor/oncogene cascade with context-dependent consequences on cell cycle progression. Mol Cell Biol 34: 2350-2358, 2014.

17. Yuan H and Gao Y: MicroRNA-1908 is upregulated in human osteosarcoma and regulates cell proliferation and migration by repressing PTEN expression. Oncol Rep 34: 2706-2714, 2015.

18. Kim HR, Shin CH, Lee H, Choi KH, Nam DH, Ohn T and Kim HH: MicroRNA-1908-5p contributes to the oncogenic function of the splicing factor SRSF3. Oncotarget 8: 8342-8355, 2017.

19. Jin JC, Jin XL, Zhang X, Piao YS and Liu SP: Effect of OSW-1 on microRNA expression profiles of hepatoma cells and functions of novel microRNAs. Mol Med Rep 7: 1831-1837, 2013.

20. Lian D, Wang ZZ and Liu NS: MicroRNA-1908 is a biomarker for poor prognosis in human osteosarcoma. Eur Rev Med Pharmacol Sci 20: 1258-1262, 2016.

21. Xia X, Li Y, Wang W, Tang F, Tan J, Sun L, Li Q, Sun L, Tang B and He S: MicroRNA-1908 functions as a glioblastoma oncogene by suppressing PTEN tumor suppressor pathway. Mol Cancer 14: 154, 2015.

22. Shou J, Gu S and Gu W: Identification of dysregulated miRNAs and their regulatory signature in glioma patients using the partial least squares method. Exp Ther Med 9: 167-171, 2015.

23. Aigner A: MicroRNAs (miRNAs) in cancer invasion and metastasis: Therapeutic approaches based on metastasis-related miRNAs. J Mol Med (Berl) 89: 445-457, 2011.

24. Mamoori A, Gopalan V, Smith RA and Lam AK: Modulatory roles of microRNAs in the regulation of different signalling pathways in large bowel cancer stem cells. Biol Cell 108: 51-64, 2016.

25. Yde CW, Sehested A, Mateu-Regué À, Østrup O, Scheie D, Nysom K, Nielsen FC and Rossing M: A new NFIA:RAF1 fusion activating the MAPK pathway in pilocytic astrocytoma. Cancer Genet 209: 440-444, 2016.

26. Wang F, Jiang C, Sun Q, Yan F, Wang L, Fu Z, Liu T and Hu F. miR-195 is a key regulator of Raf 1 in thyroid cancer. Onco Targets Ther 8: 3021-3028, 2015. 